\title{
A ciascuno il suo o il proprio?
}

\section{Paolo D'Achille}

PUBBLICATO: 12 FEBBRAIO 2019

\section{Quesito:}

Sono pervenute ulteriori richieste sulla scelta tra suo e proprio, con specifico riguardo all'espressione a ciascuno il suo/a ciascuno il proprio.

\section{A ciascuno il suo o il proprio?}

In questa espressione, dunque con riferimento al pronome indefinito ciascuno, suo e proprio sono intercambiabili, come del resto si evince già dalla risposta data a suo tempo su questo stesso sito. Possiamo aggiungere che la ricerca su Google delle due stringhe (effettuata il 25/or/2019) ha dato questo esito: 359.00o risultati per "a ciascuno il suo" e 94.000 risultati per "a ciascuno il proprio".

Il dato potrebbe essere alterato dal fatto che $A$ ciascuno il suo è il titolo di un romanzo di Leonardo Sciascia (del I966) da cui Elio Petri trasse un famoso film (del I967); molte occorrenze in rete riguardano effettivamente l'una o l'altra opera e dunque, a rigore, andrebbero defalcate dal conto.

Ma proprio la memoria del titolo potrebbe indirizzare chi parla e scrive verso suo in questo contesto e in effetti nell'uso attuale sembra di cogliere la seguente tendenza: quando nell'espressione l'aggettivo è in funzione pronominale, a ciascuno il suo è più frequente di a ciascuno il proprio. Se invece segue un nome (e dunque il possessivo resta aggettivo), la preferenza va a proprio: a ciascuno il proprio compito; a ciascuno la propria parte di responsabilità. Ma anche a ciascuno il suo destino è espressione comune.

Insomma, in questo caso il contesto lascia a chi parla o a chi scrive piena libertà di scelta tra suo e proprio e non ci sono rischi di incorrere in errore.

\section{Cita come:}

Paolo D'Achille, A ciascuno ilsuo o ilproprio?, "Italiano digitale", 2019, VIII, 2019/1 (gennaio-

marzo), pp. 20.

DOI: $10.35948 / 2532-9006 / 2019.3067$

Copyright 2019 Accademia della Crusca

Pubblicato con licenza creative commons CC BY-NC-ND 